\title{
When prognosis precedes diagnosis: putting the cart before the horse
}

\author{
Marc S. Sabatine \\ ß See related article page 1685
}

$\mathrm{E}$ ach year in the United States, some 6 million people present to emergency departments with chest pain. ${ }^{1}$ Although only $15 \%-20 \%$ of these patients are ultimately diagnosed with an acute coronary syndrome (ACS), ${ }^{2}$ about half are admitted for evaluation. Conversely, $2 \%$ of patients with ACS are mistakenly discharged. ${ }^{2}$ As patients with ACS have a relatively high risk of major adverse cardiovascular events in the short term, there is a clear need for accurate, objective tools by which to identify them.

Biomarkers can be used to aid in diagnosis and prognosis. Traditionally, diagnostic tests are used to determine whether a patient has a particular disease. In contrast, prognostic tests are used to determine the probability of a specific clinical outcome in a patient known to have the disease. Inevitably there is overlap, typically because a positive result for a diagnostic test will identify a patient as having a disease that carries a poor prognosis. The measurement of troponin concentrations in serum, for example, serves as the current "gold standard" for the diagnosis of myocardial infarction;" because it accurately identifies the presence of myocyte necrosis, the test is also used to define ACS patients at increased risk of death and ischemic complications. ${ }^{4-6}$ Nonetheless, when evaluating a potential new biomarker, it is important to consider carefully the role it may best serve and hence how it should best be judged.

One such test involves the albumin-cobalt binding assay. In the presence of ischemia, the amino-terminal end of albumin undergoes structural changes that appear to involve modifications to an aspartate-alanine-histidinelysine sequence by acidosis, low oxygen tension and free radicals. ${ }^{7}$ These changes decrease the binding capacity for cobalt, which serves as the basis for measuring ischemiamodified albumin (IMA). Elevated levels of IMA have been documented in patients experiencing myocardial ischemia in the setting of percutaneous coronary intervention. ${ }^{8-10}$ More recently, the utility of IMA measurement for the diagnosis of ACS has been tested in patients with chest pain. ${ }^{11-13}$ Using boundaries of either $85 \mathrm{U} / \mathrm{mL}$ (the 95 th percentile in healthy volunteers and the assay manufacturer's recommended upper limit of the normal range) or $90 \mathrm{U} / \mathrm{mL}$, these investigators found that IMA levels were elevated in more than $70 \%$ of patients presenting to an emergency department with chest pain, and that the test had good sensitivity (in the area of $80 \%-90 \%$ ) but poor specificity (around 30\%-50\%).

Worster and colleagues ${ }^{14}$ examined the utility of IMA using a different approach, as reported in this issue. They enrolled 189 patients who presented to the emergency department within 6 hours of the onset of potential ACS symptoms, for whom the treating physician ordered a troponin test, who had no serious cardiovascular outcome and for whom no decision to admit to hospital was made before the results of the first troponin test became available. Enrolled patients were followed for 72 hours for the occurrence of a serious cardiovascular outcome, defined as cardiovascular death, myocardial infarction, congestive heart failure, a serious arrhythmia, or refractory ischemic pain. Thus, it was the prognostic rather than the diagnostic capacity of IMA measurement that was investigated.

The results in this study were disappointing. Nearly $13 \%$ of the cohort had a serious cardiovascular outcome during the next 72 hours. When patients were stratified by baseline IMA and a cut-off value of $80 \mathrm{U} / \mathrm{mL}$ was applied, event rates were about $12 \%$ in those with high IMA levels and higher (just under 16\%) in those with low IMA concentrations. The authors calculated the positive likelihood ratio to be 0.92 (95\% confidence interval [CI] $0.69-$ 1.22 ) and the negative likelihood ratio as 1.25 (95\% CI $0.63-2.46)$. As these are prognostic data, the authors might have chosen to calculate the relative risk of an adverse outcome after a positive test result, which, based on the data, was 0.77 (95\% CI 0.34-1.74). Regardless of the metric, the message is qualitatively the same: IMA measurements do not appear to provide any useful prognostic data in this setting.

However, these results are not surprising and may be explained by taking into account the characteristics of the IMA test. Before this publication, it was already clear that IMA elevation is nonspecific and hence generates many false-positive results. The false positives will represent patients with non-ischemic chest pain, who tend to have a good prognosis and hence attenuate any statistical association between IMA elevation and serious cardiovascular outcomes. Therefore, using a nonspecific biomarker such as IMA for prognosis before first establishing diagnosis is a bit like putting the cart before the horse. 
Compounding this problem is the fact that Worster and colleagues ${ }^{14}$ opted to use $80 \mathrm{U} / \mathrm{mL}$ as the cut-off value for IMA elevation, which is lower than the manufacturer's recommendation of $85 \mathrm{U} / \mathrm{mL}$. At the $\geq 80-\mathrm{U} / \mathrm{mL}$ threshold, IMA levels were considered to be elevated in $75 \%$ of patients at presentation and in $94 \%$ at any time during the first 6 hours. Understandably, then, it would be hard for those with elevated IMA levels to be at increased risk compared to the rest of the group, when those with IMA elevations constitute almost the entire group. The authors note that when they looked into using the assay manufacturer's recommended cut-off point of $85 \mathrm{U} / \mathrm{mL}$, their study results were not appreciably different. It would have been useful to have seen not only those data but also a receiver-operatingcharacteristic curve analysis.

In conclusion, the study by Worster and colleagues reinforces the fact that IMA elevation is relatively nonspecific and is thus unlikely to help identify a subgroup of patients at high risk for serious adverse cardiovascular events. However, IMA levels may still prove useful to clinicians in 2 situations. First, in terms of diagnosis, the high sensitivity of the IMA test has excellent negative predictive value; IMA measurements could thus be used to help rule out ischemia in patients with chest pain. However, since more than $70 \%$ of patients who present to the emergency department have an IMA level above the manufacturer's suggested cut-off value, the application of current thresholds would aid in the exclusion of ischemia in only a minority of patients. Higher thresholds would remedy this problem, but may erode the test's negative predictive value and thus will require further investigation. Second, measuring serum IMA levels may provide important prognostic information in patients diagnosed with unstable angina, who (by definition) have normal troponin measurements.

A reliable marker of myocardial ischemia remains an important unmet need in cardiology. ${ }^{15}$ Whether the measurement of IMA will fill this need, only time, and additional large, well-designed studies, will tell.

\section{This article has been peer reviewed.}

Dr. Sabatine is from the TIMI [Thrombolysis in Myocardial Infarction] Study Group, Cardiovascular Division, Brigham and Women's Hospital, Boston, Mass.

Competing interests: None declared.

\section{References}

1. McCaig LF, Burt CW. National Hospital Ambulatory Medical Care Survey: 2002 Emergency Department summary. Advance data from Vital and Health Statistics. Hyattsville, MD: National Center for Health Statistics; 2004.

2. Pope JH, Aufderheide TP, Ruthazer R, Woolard RH, Feldman JA, Beshansky JR, et al. Missed diagnoses of acute cardiac ischemia in the emergency department. N Engl 7 Med 2000;342(16):1163-70.

3. Jaffe AS, Ravkilde J, Roberts R, Naslund U, Apple FS, Galvani M, et al. It's time for a change to a troponin standard. Circulation 2000;102(11):1216-20.

4. Antman EM, Tanasijevic MJ, Thompson B, Schactman M, McCabe CH, Cannon CP, et al. Cardiac-specific troponin I levels to predict the risk of mortality in patients with acute coronary syndromes. New Engl 7 Med 1996;335:1342-9.

5. Morrow DA, Cannon CP, Rifai N, Frey MJ, Vicari R, Lakkis N, et al. Ability of minor elevations of troponins I and $\mathrm{T}$ to predict benefit from an early invasive strategy in patients with unstable angina and non-ST elevation myocardial infarction: results from a randomized trial. 7AMA 2001;286:2405-12.

6. Braunwald E, Antman EM, Beasley JW, Califf RM, Cheitlin MD, Hochman JS, et al. ACC/AHA guideline update for the management of patients with unstable angina and non-ST-segment elevation myocardial infarction - 2002: summary article: a report of the American College of Cardiology/American Heart Association Task Force on Practice Guidelines (Committee on the Management of Patients With Unstable Angina). Circulation 2002;106(14):1893-900.

7. Bar-Or D, Curtis G, Rao N, Bampos N, Lau E. Characterization of the $\mathrm{Co}^{2+}$ and $\mathrm{Ni}^{2+}$ binding amino-acid residues of the $\mathrm{N}$-terminus of human albumin: an insight into the mechanism of a new assay for myocardial ischemia. Eur 7 Biochem 2001;268:42-7.

8. Bar-Or D, Winkler JV, Vanbenthuysen K, Harris L, Lau E, Hetzel FW. Reduced albumin-cobalt binding with transient myocardial ischemia after elective percutaneous transluminal coronary angioplasty: a preliminary comparison to creatine kinase-MB, myoglobin, and troponin I. Am Heart 7 2001;141:985-91.

9. Sinha MK, Gaze DC, Tippins JR, Collinson PO, Kaski JC. Ischemia modified albumin is a sensitive marker of myocardial ischemia after percutaneous coronary intervention. Circulation 2003;107:2403-5.

10. Quiles J, Roy D, Gaze D, Garrido IP, Avanzas P, Sinha M, et al. Relation of ischemia-modified albumin (IMA) levels following elective angioplasty for stable angina pectoris to duration of balloon-induced myocardial ischemia. Am 7 Cardiol 2003;92:322-4.

11. Sinha MK, Roy D, Gaze DC, Collinson PO, Kaski JC. Role of "ischemia modified albumin", a new biochemical marker of myocardial ischaemia, in the early diagnosis of acute coronary syndromes. Emerg Med 7 2004;21:29-34.

12. Roy D, Quiles J, Aldama G, Sinha M, Avanzas P, Arroyo-Espliguero R, et al Ischemia modified albumin for the assessment of patients presenting to the emergency department with acute chest pain but normal or non-diagnostic 12-lead electrocardiograms and negative cardiac troponin T. Int 7 Cardiol 2004; $97: 297-301$.

13. Anwaruddin S, Januzzi JL Jr, Baggish AL, Lewandrowski EL, Lewandrowski $\mathrm{KB}$. Ischemia-modified albumin improves the usefulness of standard cardiac biomarkers for the diagnosis of myocardial ischemia in the emergency department setting. Am 7 Clin Pathol 2005;123:140-5.

14. Worster A, Devereaux PJ, Heels-Ansdell D, Guyatt GH, Opie J, Mookadam $\mathrm{F}$, et al. Capability of ischemia-modified albumin to predict serious cardiac outcomes in the short term among patients with potential acute coronary syndrome. CMA7 2005;172(13):1685-90.

15. Morrow DA, de Lemos JA, Sabatine MS, Antman EM. The search for a biomarker of cardiac ischemia [editorial]. Clin Chem 2003;49:537-9.

Correspondence to: Dr. Marc S. Sabatine, Cardiovascular Division, Brigham and Women's Hospital, 75 Francis St., Boston MA 02115; fax 617 734-7329; msabatine@partners.org 